\title{
A general rotation averaging method for granular materials
}

\author{
Jia $\operatorname{Lin}^{1} \cdot$ Wei Wu ${ }^{1}$
}

Received: 10 June 2016 / Published online: 24 May 2017

(C) The Author(s) 2017. This article is an open access publication

\begin{abstract}
In order to study the rotational behavior of granular materials, rotation averaging methods are required. Most of the existing rotation averaging methods are only for $2 \mathrm{D}$ cases and limited by the shape, position and size of the averaging volumes. Hence, a general rotation averaging method is proposed in this paper. Our approach is simple yet works for both $2 \mathrm{D}$ and $3 \mathrm{D}$ cases with very little restriction. The performance of this method is shown by both hypothetical examples and DEM simulations of plane strain tests with different boundary conditions.
\end{abstract}

Keywords Rotation averaging · Granular materials · DEM

\section{Introduction}

The overall mechanical behaviors of granular materials can be reasonably well described by continuum approaches $[3,9,11,22,23,29]$. Since granular materials are discrete in nature, some attempts have been made to obtain the continuum properties by averaging methods $[2,5,6,16,25,39]$, which are also referred as homogenization methods $[7,19]$ or coarse grain methods $[30,38]$. A meso-scaled averaging volume which contains several particles needs to be defined for an averaging method. The overall behaviors of these particles are averaged by certain mathematical approaches. There are several kinds of averaging methods to obtain different continuum properties from the granular materials, such as the void ratio, stress, strain, fabric tensor and rotation.

\section{Jia Lin}

jia.lin@boku.ac.at

1 Institution of Geotechnical Engineering, University of Natural Resources and Life Sciences, Vienna, Vienna, Austria
Particle rotations and the associated frictional work play an important role in understanding the behavior of granular materials. Therefore, if granular materials need to be accurately described, a model is needed to describe the discrete rotation of each point as well as its translation. Micropolar continuum theory, which is also known as Cosserat theory, is an enhanced continuum theory which takes into account the independent rotations inside the material. An important assumption of micropolar theory is that the rotation of individual material point can be different from the continuum rotation. For granular materials, as can be seen in experiments or DEM simulations, the particles in the shear band usually show larger rotations than those outside the shear band. Moreover, the particle rotations are not equal to the rotations in continuum mechanics. Another assumption related to the rotation in micropolar continuum is that the stress tensor is asymmetric $[12,28,40]$. By using micropolar continuum, several researches $[1,17,26,27,31-36]$ have shown that some salient features of the granular materials can be well modeled. However, a direct comparison of the micropolar rotation with discrete rotations is not feasible. Hence, a rotational averaging method is needed to obtain continuum rotation from the translation and rotation of discrete particles.

Some examples of micropolar homogenization for 2D granular material systems are given by Kruyt [19] and Ehlers et al. [7]. Kruyt presented a theoretical framework of discrete Cosserat (micropolar)-type granular materials for both static and kinematic cases. The homogenization method of Ehlers considers the particles on the boundary of the homogenization volume. The optimized averaging area is found to be about five times of the mean grain diameter. In [13,20,21], an averaging method for the rotational degree of freedom is given. The method is realized by summing up all the rotations within the averaging volume weighted by the volume of particles. A general averaging method is proposed 
by Zhu et al. [42] for particle-fluid flow in a gas fluidized bed.

However, the averaging methods above are very complicated and depend highly on the shape, position and scale of the averaging volumes $[7,10]$. Some methods do not take the positions of particles into account, which is not correct since particles in different positions (center or boundary of the averaging volume) have different contributions on the overall rotation. A new method of rotation averaging for granular material is proposed here. This averaging method is simple yet works for both $2 \mathrm{D}$ and $3 \mathrm{D}$ cases. It is not restricted by the size or position of the averaging volume. The method is shown for granular material with circular shaped particles (discs in 2D and spheres in 3D). However, it can be easily applied to arbitrary shaped particles.

\section{Rotation averaging method}

According to Stokes' theorem, the integral over the boundary of some orientable manifold is equal to the integral of its exterior derivative over the whole manifold. Similarly, in averaging method, it is equivalent to consider all the elements inside the averaging volume $[19,21,42]$ or to consider all the elements in the averaging boundary $[7,8]$. The method proposed here considers the elements in the averaging boundary, which is similar to the averaging method used by Ehlers et al. [7]. However, the averaging volume is not formed by connecting the centers of all boundary particles in our approach. A sphere or circle shaped averaging volume is used for rotation averaging in 3D or 2D cases. The general steps of the averaging method are shown in the following:

First, an averaging circle needs to be defined (an averaging sphere in 3D case), the center and radius of the averaging circle can be chosen arbitrarily. All the particles which intersect with this circle needs to be identified. If the radius, position, velocity and rotational velocity of these particles are known, the rotational velocity of the averaging circle can be calculated.

If we connect the particle centers and the center of the averaging volume with straight lines and extend these lines to the particle boundary, the intersect points between these lines and the averaging circle (or sphere) can be find out. By knowing velocity and rotation of a particle, the velocity of a intersect point can be calculated by:

$\mathbf{v}_{i}=\mathbf{v}_{p}+\omega_{p} \times \mathbf{r}_{p i}$

where $\mathbf{v}_{i}$ is the velocity of the intersect point, $\mathbf{v}_{p}$ is the velocity of the particle center, $\mathbf{r}_{p i}$ is the vector from the particle center to the intersect point and $\omega_{p}$ is the rotational velocity of the particle (the rotational center of $\omega_{p}$ is the center of the particle).
The the velocity $\mathbf{v}_{i}$ leads to a rotational velocity of the averaging circle $\left(\omega_{i}\right)$, the rotational cener of $\omega_{i}$ is the center of the averaging circle.

$\omega_{i}=\frac{\mathbf{r}_{a} \times \mathbf{v}_{i}}{\left\|\mathbf{r}_{a}\right\|^{2}}$

where $\mathbf{r}_{a}$ is the vector connecting the center of the averaging circle and the intersect point.

By summing up all the $\omega_{i}$, the rotational velocity of the averaging circle can be obtained. For 2D case, the averaged rotational velocity is:

$\omega_{a}=\frac{1}{2 \pi} \sum \alpha_{i} \omega_{i}$

where $\alpha_{i}$ is the angle of the intersecting arc on the averaging circle. In this way, the velocity of each intersect point is weighted by the arc length on the averaging circle.

For 3D case, the averaged rotational velocity is:

$\omega_{a}=\sum \frac{1}{2}\left(1-\cos \frac{\alpha_{i}}{2}\right) \omega_{i}$

since the area of the spherical cap equals to $2 \pi\left\|\mathbf{r}_{a}\right\|^{2}(1-$ $\left.\cos \left(\alpha_{i} / 2\right)\right)$, while the area of the whole sphere is $4 \pi\left\|\mathbf{r}_{a}\right\|^{2}$. Here, $\alpha_{i}$ is a conical angle of the projection of the spherical particle on the projection sphere. The velocity of each intersect point is weighted by the area on the averaging sphere. For arbitrary shaped averaging volume, the determination of surface area becomes very complicated and will not be discussed here.

Figure 1 shows an example of the averaging method in 2D, the small circles in blue are particles and the large circle in yellow is the averaging circle. For each particle, their velocities are shown, the particle velocity in the particle center $\mathbf{v}_{p}$

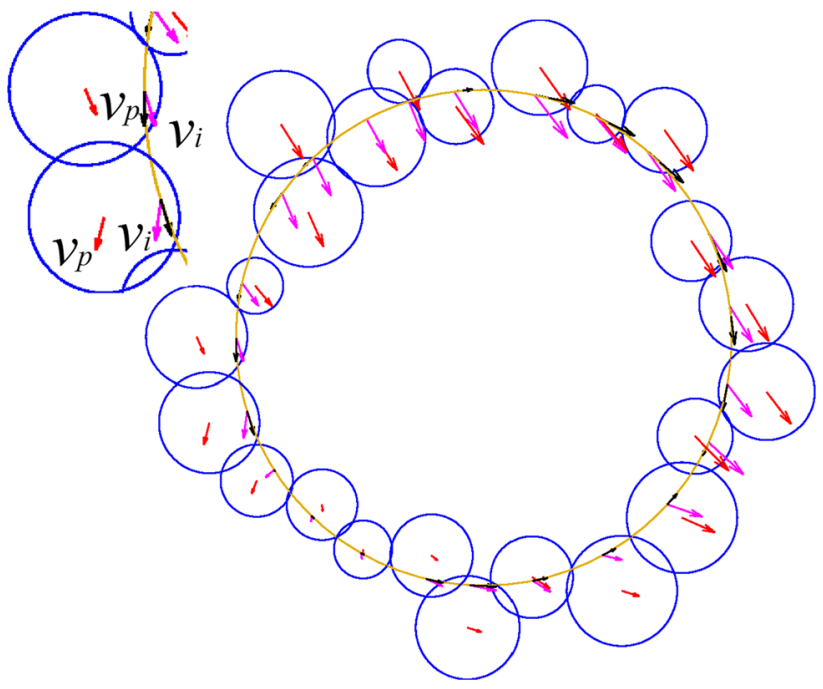

Fig. 1 Rotation averaging in 2D 


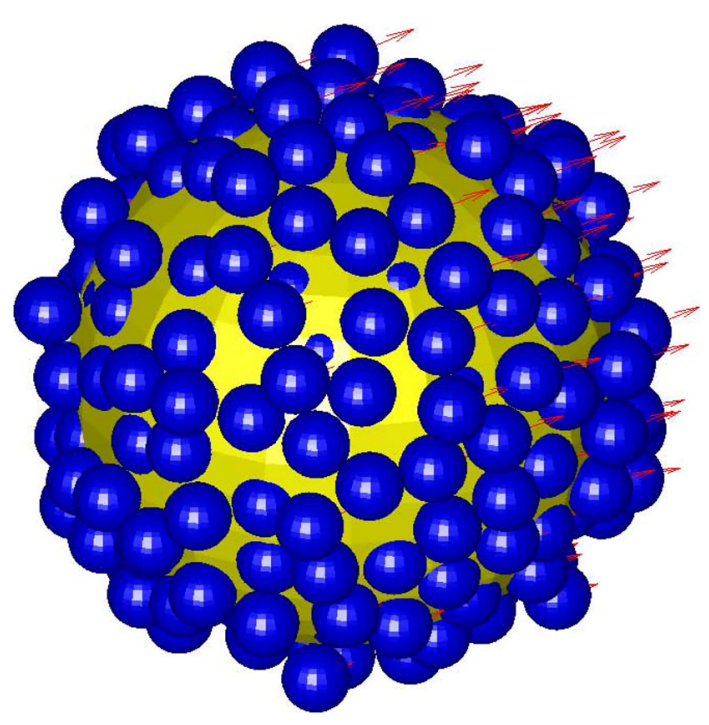

Fig. 2 Rotation averaging in 3D

(red), the velocity at the intersect point $\mathbf{v}_{i}$ (magenta) and the tangential projection of $\mathbf{v}_{i}$ on the averaging circle (black). By summing up all the tangential projection velocities, the rotation of the averaging circle can be obtained.

Similarly, an example of 3D rotation averaging is shown in Fig. 2, where the blue spheres are particles and the yellow sphere is the averaging sphere. Velocities of particles are shown by red arrows. It can be noticed that in $2 \mathrm{D}$ case, almost the whole averaging circle is intersecting with particles while in 3D case, only a part of it has intersections with particles. From our calculations, about $60 \%$ of the averaging sphere is intersecting with particles in $3 \mathrm{D}$ case, while in 2D case it is about $100 \%$. This results are not very much dependent on the specimen construction method, i.e. particle packing method. Even for very loose packing in 2D case, the intersection percentage is still close to $100 \%$. For 3D case, the difference between dense and loose packing are not very large, but if particles with different diameters are used, the intersection percentage will increase, since some pores are filled by small particles.

For other shape of averaging volumes, this method can still be used. Finding out $\mathbf{v}_{i}$ for each intersection point is the same. However, $\mathbf{r}_{a}$ should be different for each intersection points. Also, we need to calculate circumference for $2 \mathrm{D}$ averaging or surface area for 3D averaging and the weighting factor of each particle, which can be very complicated for nonspherical averaging volumes.

\section{Hypothetical examples}

Two hypothetical examples are used to show the performance of the averaging method. First, a simple example containing

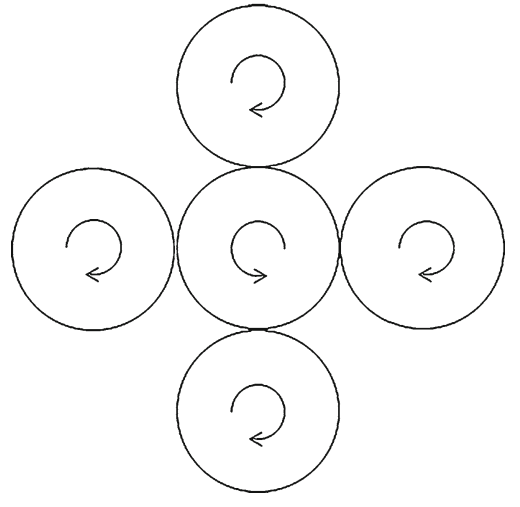

Fig. 3 A 2D example for rotation averaging

only 5 particles are shown with different scales of averaging volumes. In Fig. 3, there are five particles with no translational velocity. The center particle rotates counter-clockwise and other particles rotate clockwise. The all particles have the same radius $r$ and rotational speed $\omega$. Hence, on the contact points, there are only relative rolling without any slipping between particles. The example is similar as gears in a planetary gearbox system.

The center of the averaging circle is chosen to be coincide with the center of particle in the middle, and the radius of the averaging circle is $R$. If $R \leq r$ as shown in Fig. $4 \mathrm{a}$, there is only one intersect point and the intersecting arc length is $2 \pi R$. Hence, the averaged rotation equals to the particle rotation. If $r<R<2 r$ (Fig. 4b), there are four intersect points. The averaged rotation is counter-clockwise. For the
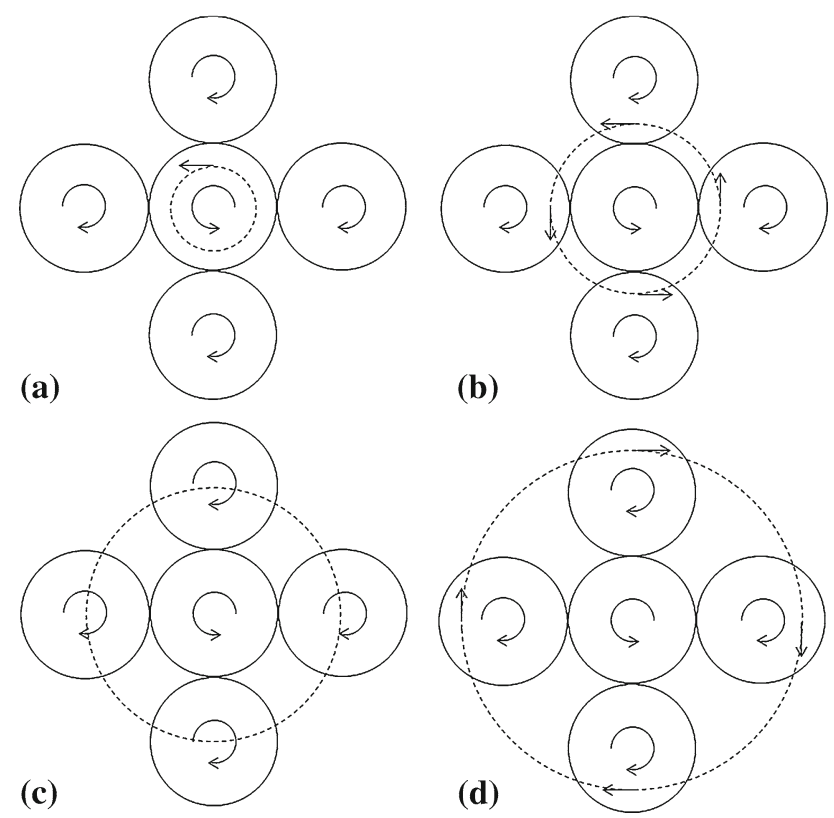

Fig. 4 Differnet rotation averaging results depending on the size of the averaging volumes, a $R<r, \mathbf{b} r<R<2 r, \mathbf{c} R=2 r, \mathbf{d} 2 r<R<3 r$ 
case $R=2 r$ (Fig. 4c), the intersect velocities are zero, so the averaged rotation is also zero, which means all the rotations canceled out within the averaging volume. For $R>2 r$ in Fig. $4 d$, the averaged rotation is clockwise. Note that this is just a hypothetical example, in which the particles have no translational speed and very large rotations. In reality, the averaged rotation will not change so much for different averaging volume sizes.

For the second example, let us consider a plane strain test with a shear band in the middle, as shown in Fig. 5. The size of particles are small enough to be neglected in this example. Therefore, we only consider the translation speeds of particles. If the averaging circle does not intersect with the shear band, the averaged rotation is zero. For example, the averaging circle on the top, all particles intersect with this averaging circle have the same velocity, so the rotations canceled out. If the averaging circle intersect with the shear band, like the averaging circle in the middle, the averaged rotation is non zero.

These two hypothetical examples show that the results obtained by the averaging method are qualitatively correct. However, we have to make some assumptions and neglect the translational or rotational speed of individual particles in these examples, which makes these two examples far from the reality. Hence, discrete element simulations are carried out in the next section to test the performance of the averaging method.

\section{Discrete element method simulations}

Discrete element methods (DEM) have been widely used to study the rotational behavior in granular materials $[4,14,18$,

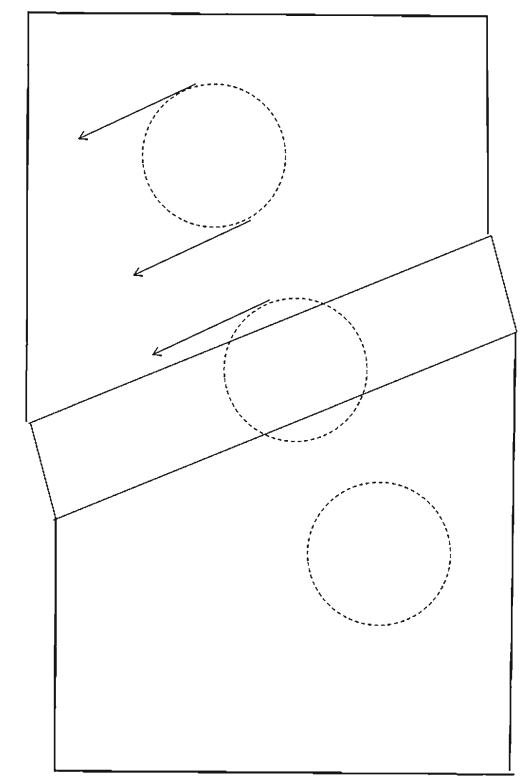

Fig. 5 Rotation averaging of a plane strain test
$24,27,37,39]$. In order to apply the rotation averaging method on discrete particles, two DEM simulations of plane strain tests with different boundary conditions are carried out. The DEM simulations follow three general steps: model generation, isotropic loading and biaxial loading. The commercial DEM software package PFC 3D is used here. In PFC 3D, there are two kinds of elements: ball and wall. Ball elements are used to model discrete particles and the wall elements are used to apply boundary conditions.

First, six walls are generated to apply boundary conditions for the plane strain test, two fixed walls with a distance of $20 \mathrm{~mm}$ in the out-of-plane (Y) direction and four walls in $\mathrm{X}$ and $\mathrm{Z}$ directions, see Fig. 6 . The size of the model is $40 \mathrm{~mm}$ $\times 20 \mathrm{~mm} \times 20 \mathrm{~mm}$. Then, balls are generated within the area between the walls to model granular materials. For the generation of balls, the radius expansion method is used. First, balls with smaller radius are randomly generated in the space. Then, the radius of balls are expanded to obtain the desired void ratio. Due to the radius expansion, there are big overlaps between balls. In order to avoid this, several steps of calculations are carried out until a statical state is reached. During the generation of balls, all the walls remain stationary. In this model, there are 150,000 balls with a void ratio of 0.67 . Radius of balls are distributed linearly, the ratio of largest to smallest ball radii is $3: 2$, the average radius of particles is $0.244 \mathrm{~mm}$. The friction coefficient on the ball surface is 0.577 .

The second step is isotropic loading in which the model is loaded to an isotropic stress state in $\mathrm{X}$ and $\mathrm{Z}$ directions. This is realized by a servo mechanism. If the wall stress is larger than the required stress, the wall will move away from the balls, if the wall stress is smaller than the required stress, the wall will move towards the balls, see Eq. (5).

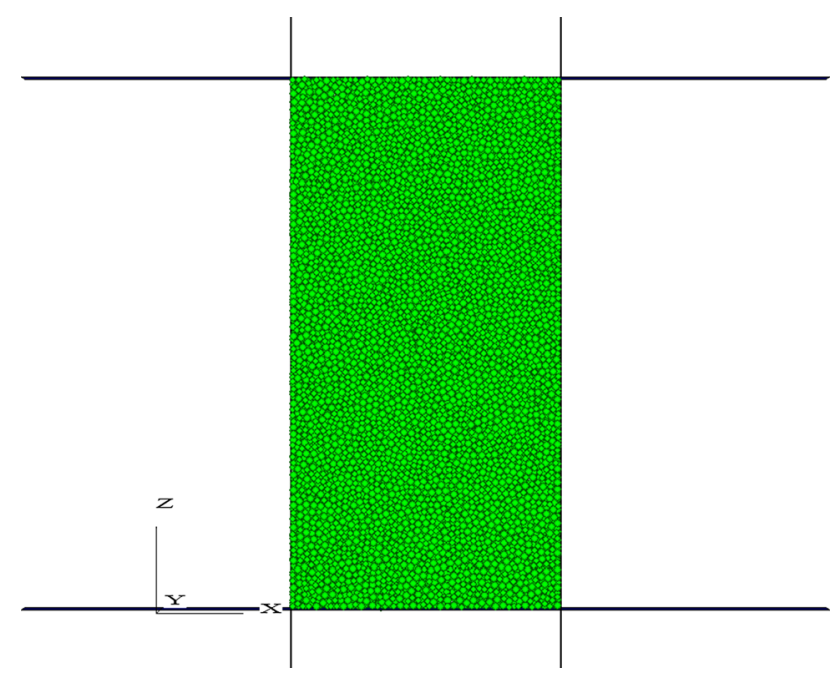

Fig. 6 DEM model of biaxial test, initial model generation 


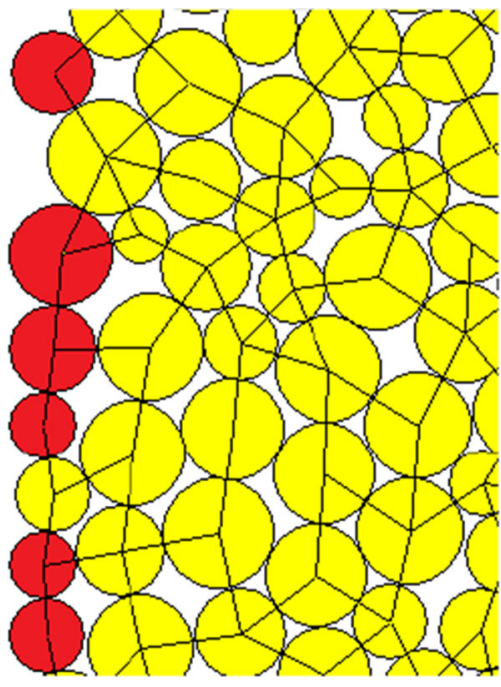

Fig. 7 Flexible stress boundary condition

$\Delta r=\frac{\alpha A}{k_{n}^{\text {wall }}}\left(\sigma^{\text {wall }}-\sigma^{\text {req }}\right)$

where $\Delta r$ is the wall displacement in one step, $A$ is the wall area, $k_{n}^{\text {wall }}$ is the contact stiffness on the wall, $\sigma^{\text {wall }}$ and $\sigma^{\text {req }}$ are the wall stress and the required stress, $\alpha$ is a relaxation factor between 0 and $1 . k_{n}^{\text {wall }}$ is calculated by summing the contact stiffness of all the balls in contact with the wall. In this way, the model can be loaded to the required isotropic stress $(6000 \mathrm{kPa}$ is used in this simulation), which is the starting point of biaxial loading.

During the biaxial loading, the lower wall is fixed and the upper wall moves down with a constant speed, while the stresses in the horizontal direction stay constant. Hence, stress boundary conditions need to be applied in X direction. Both rigid and flexible stress boundary conditions are used here. Rigid stress boundary condition can be realized by applying the servo mechanism [Eq. (5)] on the two walls perpendicular to $\mathrm{X}$ direction.

The same model with flexible stress boundary conditions are also simulated. The flexible stress boundary condition is applied through the following steps. First, the balls on the boundaries are distinguished from other balls. This is realized by going through all the contacts on each ball, if all the contact points are in the inside half of the ball, the ball is considered as a ball on the boundary. For example in Fig. 7 all the boundary balls are shown with red color.

Then, a force pointing towards the center of the model is applied on the boundary ball. The magnitude of the force depends on the radius of the ball.

$$
F_{b}=P \pi r^{2}
$$

where $F_{b}$ is the force applied on the boundary ball, $P$ is the required pressure of the stress boundary conditions and $r$ is the radius of the ball. In this way, required pressures are applied to all the balls on the boundaries.

Founding boundary balls and applying load is carried out in each step of the DEM simulation, to make sure that the balls on the boundaries can be correctly found and the loading can be correctly applied in each time step. Since the stress boundary is applied separately on each ball, large deformations in the radial direction are allowed.

For both stress boundary conditions, the model is loaded until shear bands can be clearly observed. The results of rigid boundary condition are shown in Fig. 8 and the results of flexible boundary condition are shown in Fig. 9. Colors are used to show the rotational speeds in $\mathrm{Y}$ direction of each particles. Red and yellow show high and medium rotational speed in clockwise direction, magenta and blue show high
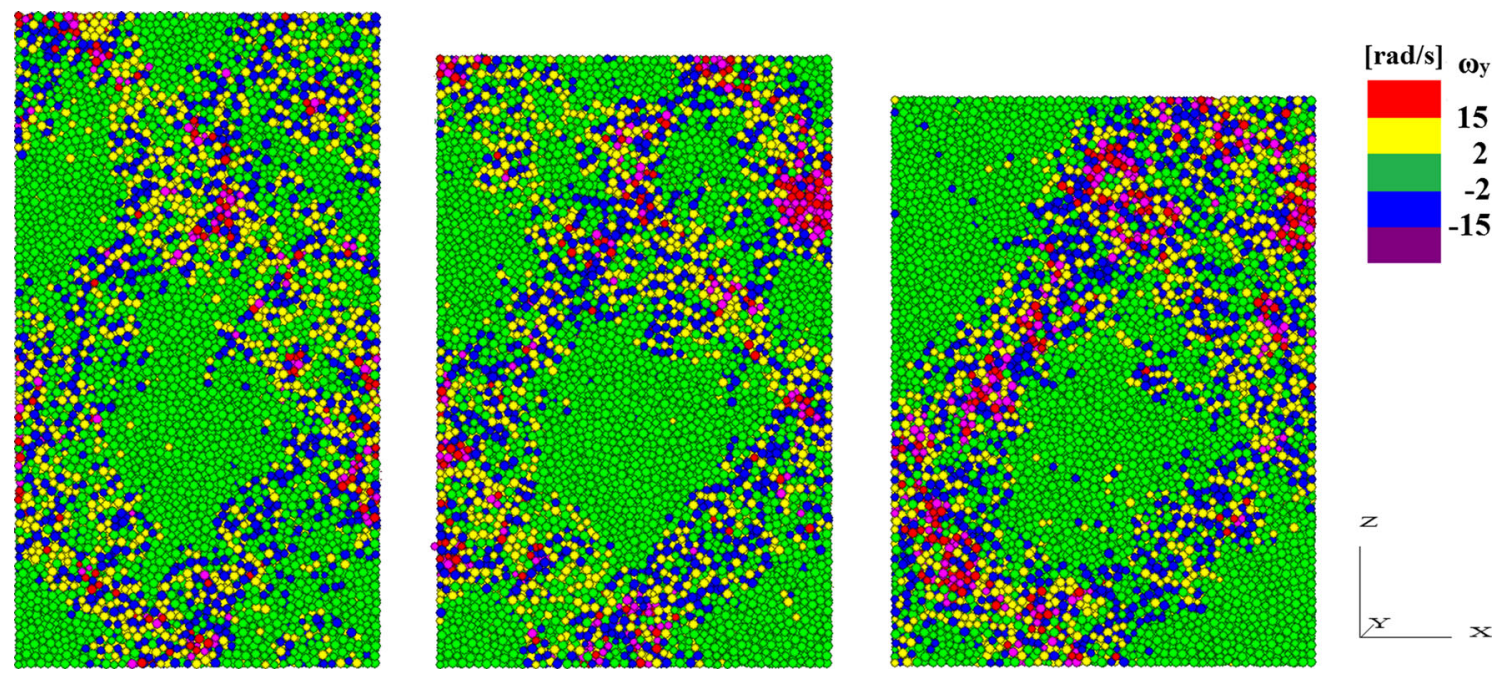

Fig. 8 Biaxial test simulation with rigid stress boundary condition, vertical strain $6.47,11.4$ and $15.8 \%$ 
and medium rotational speed in counterclockwise direction, while green means rotational speed is equal to or close to zero.

It can be seen that for both boundary conditions, shear bands appear in the simulations. The model with rigid stress boundary condition have several shear bands which conjugate each other at the boundaries. The model with flexible stress boundary condition gives one main shear band with much larger particle rotations. However, these shear bands are so far only shown by discrete rotations of individual particles, averaged rotations will be shown and discussed in the next section.

\section{Rotation averaging results}

The rotation averaging method shown above is applied to the DEM models in the last section. As discussed before, the method is not restricted by the position and size of averaging volume. Hence, it is applied to different positions of the DEM models with different diameters of the averaging sphere. In order to avoid averaging spheres outside the material boundary, only the center part of the model (size $20 \mathrm{~mm} \times 10 \mathrm{~mm}$ ) is used for rotation averaging, as shown in Fig. 10.

Taking the mean diameter of particles $d_{50}$ as a reference, the diameters of averaging sphere from $4 d_{50}$ to $12 d_{50}$ are used. The mean diameter of particles is $0.488 \mathrm{~mm}$, therefore the diameters of averaging spheres range from 1.952 to $5.856 \mathrm{~mm}$. The center points of the averaging spheres do not need to coincide with particle center points or any other particular points. Hence, the averaging method is carried out in the middle of plane strain direction $(\mathrm{y}=10 \mathrm{~mm})$ for every $1 \mathrm{~mm}$ in $\mathrm{x}$ and $\mathrm{z}$ direction, which is about twice the mean particle diameters. Therefore, $11 \times 21=231$ averaging spheres are used each time. The positions of averaging spheres are shown in Fig. 11.

For each point, the averaged rotation in $\mathrm{x}, \mathrm{y}$ and $\mathrm{z}$ direction can be obtained by the rotation averaging method. Since plane strain problems are considered here, the rotation in $\mathrm{y}$
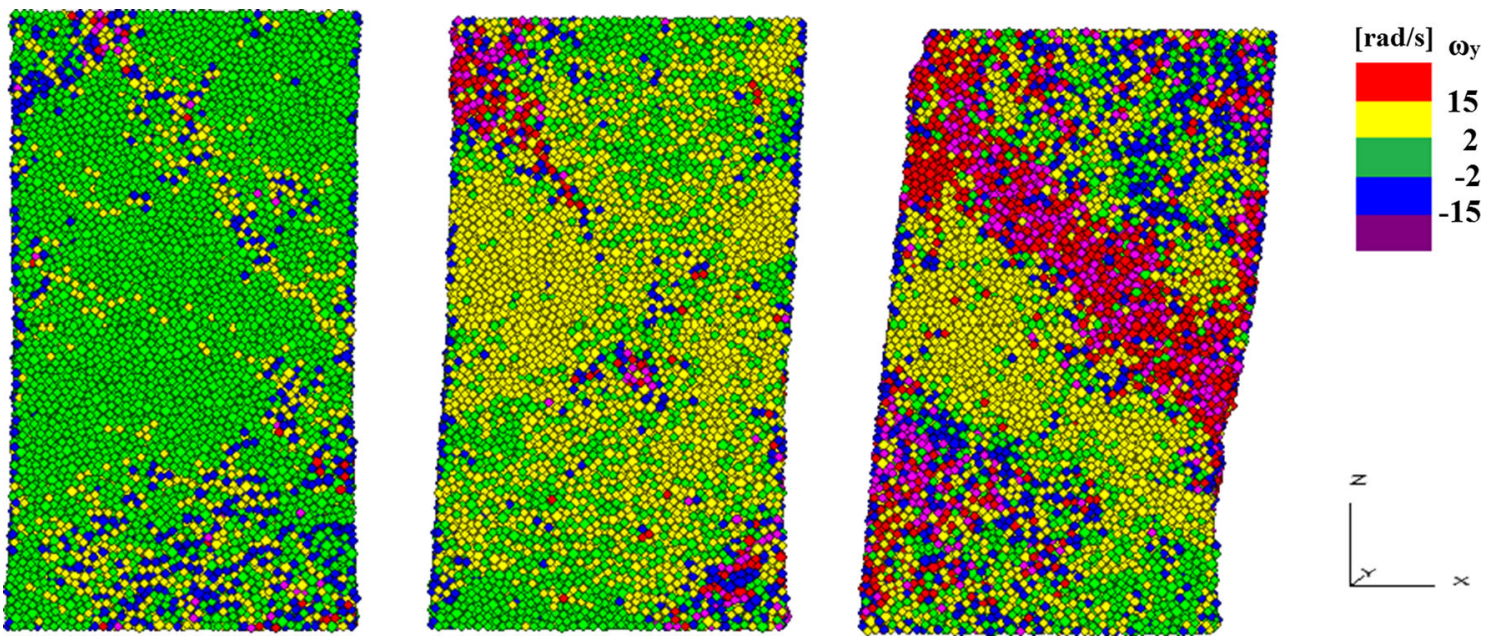

Fig. 9 Biaxial test simulation with flexible stress boundary condition, vertical strain 8.87, 9.75 and $10.6 \%$
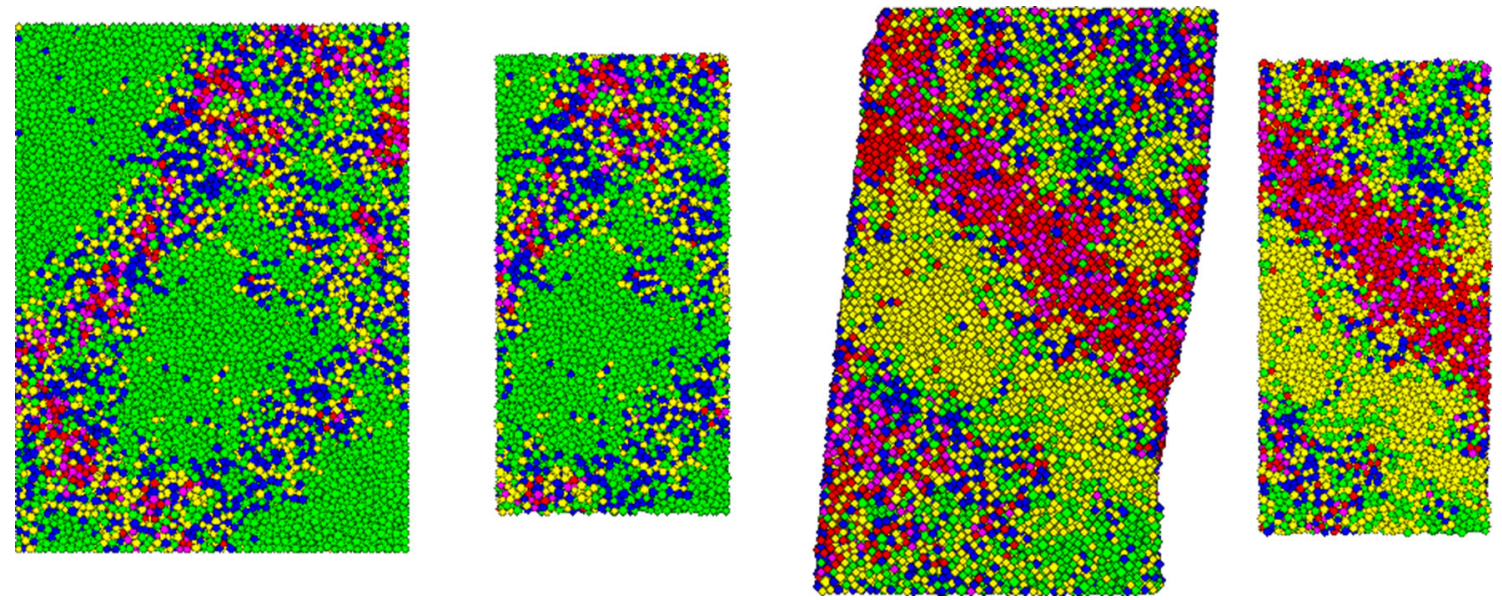

Fig. 10 Part of the DEM model used for rotation averaging 

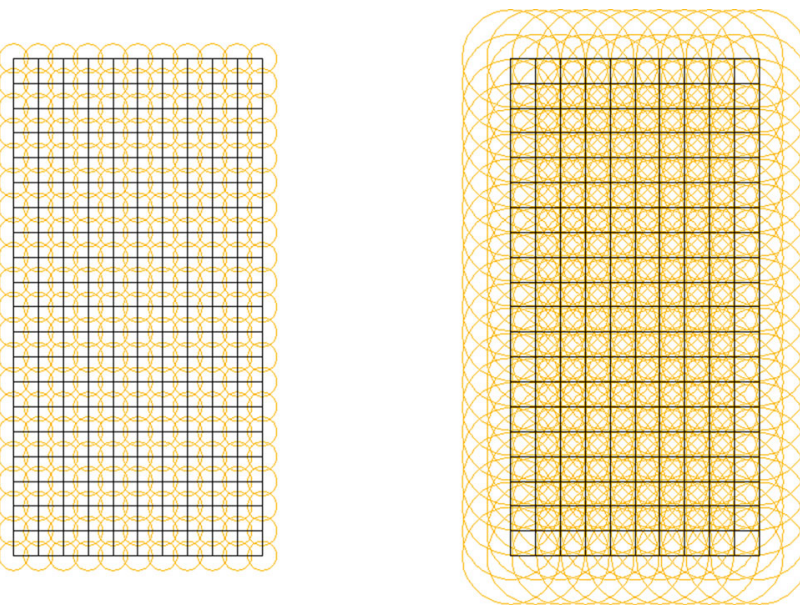

Fig. 11 Positions of averaging spheres with diameters of $4 d_{50}$ and $12 d_{50}$

direction is the most interest. The rotational speeds in $\mathrm{x}$ and $\mathrm{z}$ directions are close to zero as will be shown in next section. Hence, only the results of averaged rotational velocity in $y$ direction for both DEM simulations are plotted in Figs. 12 and 13 . In order to see the differences between the averaging results, different color scales are used.

For both models, it can be seen that the rotational behavior can be well captured by the averaging method. From the averaged results, shear bands can be clearly distinguished from the rest part of the model due to the large rotations. In Fig. 12, all four shear bands in different directions can be seen from the averaging results. The rotational speed of the shear band in the upper left corner clearly has larger rotation than other shear bands.

Regardless of the relative positions between the averaging volume and particles, the method is able to give a result of averaged rotational speed. For large averaging volumes, the overall rotational behavior in the volume covered by the averaging sphere can be obtained. While small averaging spheres give the localized rotational behaviors. It means that researchers can choose the position and size of the averaging volumes freely depending on the problem and the scale they need.

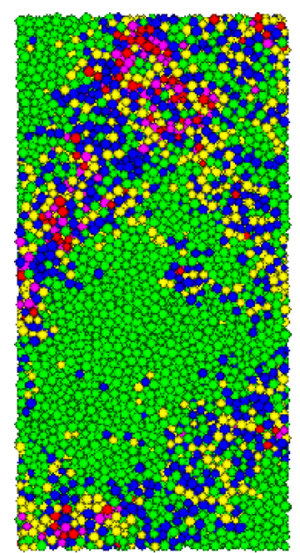

(a)

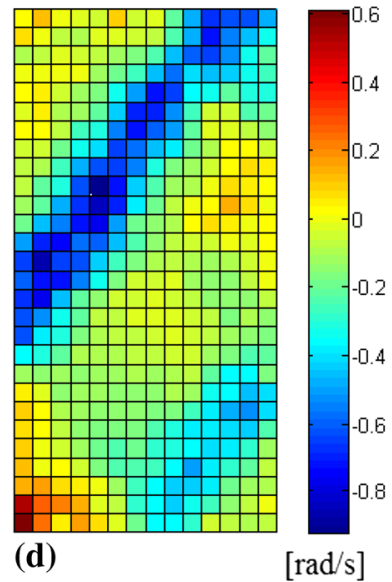

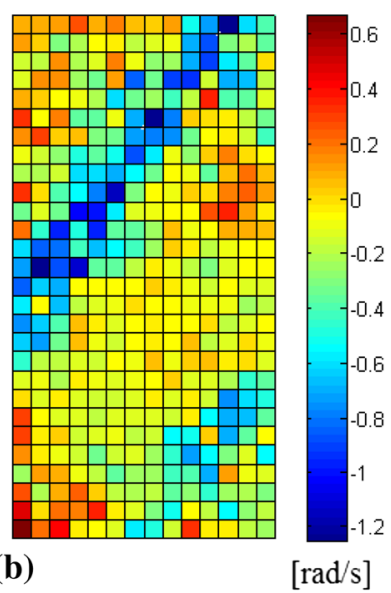

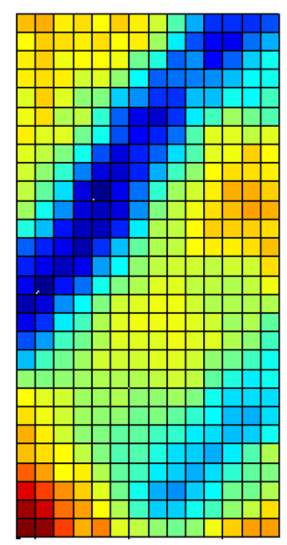

(e)

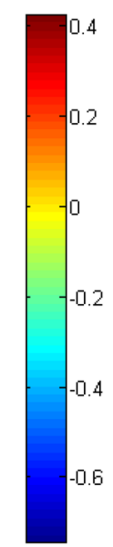

$[\mathrm{rad} / \mathrm{s}]$

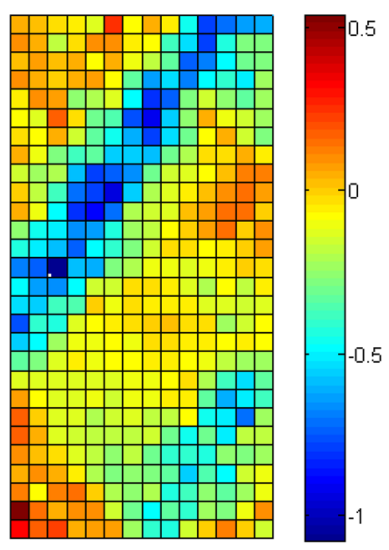

(c)

$[\mathrm{rad} / \mathrm{s}]$

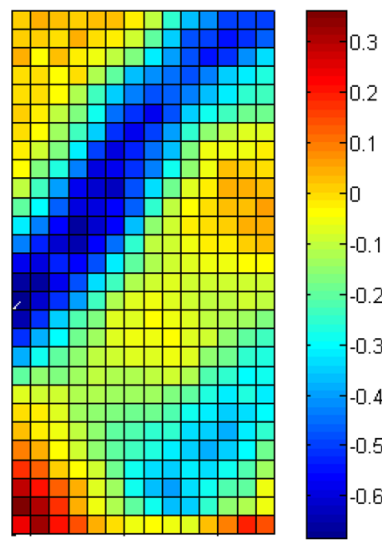

(f)

$[\mathrm{rad} / \mathrm{s}]$

Fig. 12 a DEM results. b-f Rotation averaging results with different averaging diameters: $4 d_{50}, 6 d_{50}, 8 d_{50}, 10 d_{50}$ and $12 d_{50}$, colorbars show the rotational speed in clockwise direction, model with fixed boundary conditions (color figure online) 


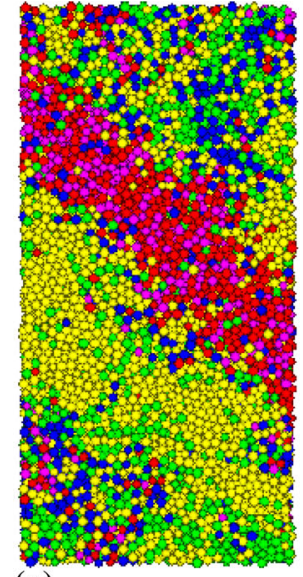

(a)

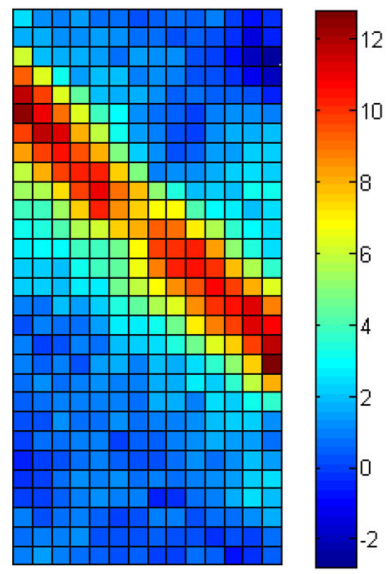

(d)

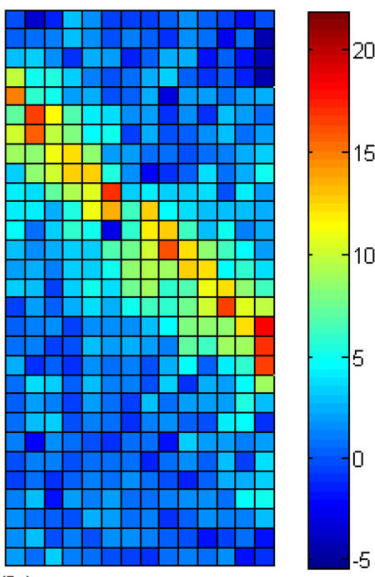

(b)

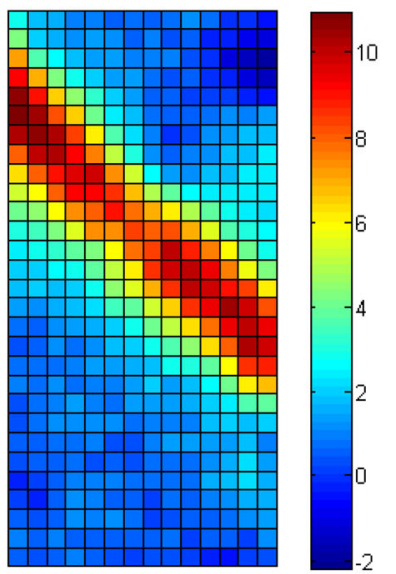

(e) $[\mathrm{rad} / \mathrm{s}]$

$[\mathrm{rad} / \mathrm{s}]$

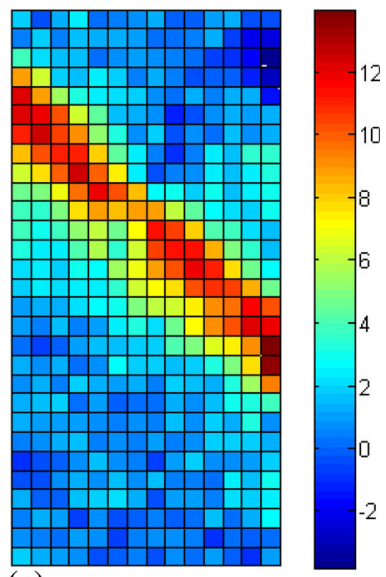

(c)

$[\mathrm{rad} / \mathrm{s}]$

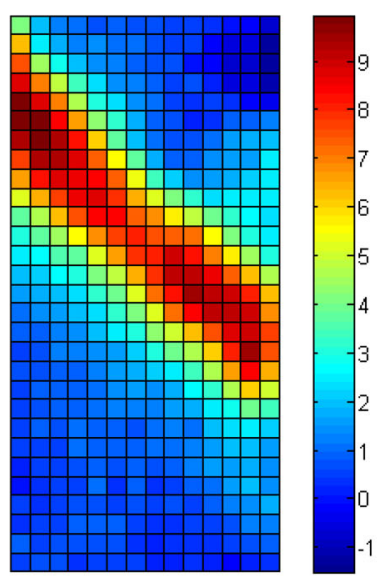

(f)

$[\mathrm{rad} / \mathrm{s}]$

Fig. 13 a DEM results. b-f Rotation averaging results with different averaging diameters: $4 d_{50}, 6 d_{50}, 8 d_{50}, 10 d_{50}$ and $12 d_{50}$, colorbars show the rotational speed in clockwise direction, model with flexible boundary conditions (color figure online)

The rotational speed obtained with the averaging should be the total spin of the material. If micropolar theory is considered, total spin equals to micropolar spin plus the vorticity. Vorticity (or macroscopic spin) is defined by:

$w:=\frac{1}{2}(\vec{\nabla} \boldsymbol{v}-\boldsymbol{v} \vec{\nabla})$

where $v$ is the velocity.

According to Eq. (1), the velocity $\mathbf{v}_{i}$ depends on $\mathbf{v}_{p}$ and $\omega_{p} \times \mathbf{r}_{p i}$. If these two parts are separated, we can get two averaged rotations, the sum of these two averaged rotations is the averaged rotation of the general method. The contributions of $\mathbf{v}_{p}$ and $\omega_{p} \times \mathbf{r}_{p i}$ can be quantified. The averaged results of all 435 points in Fig. 12b are taken. The norm of the averaged rotational velocity depending on $\mathbf{v}_{i}, \mathbf{v}_{p}$ and $\omega_{p} \times \mathbf{r}_{p i}$ are calculated. Taken rotational velocities depending on $\mathbf{v}_{i}$ to be $100 \%$, the percentages of rotational velocities depending on $\omega_{p} \times \mathbf{r}_{p i}$ are shown in Fig. 14 .
It can be seen that the percentage of $\omega_{p} \times \mathbf{r}_{p i}$ vary a lot for different points. The contribution of $\omega_{p} \times \mathbf{r}_{p i}$ are small for most of the averaging points, but it can also be more than $\pm 80 \%$ in some extreme cases. Therefore, it is meaningless to separate the contributions of $\mathbf{v}_{p}$ and $\omega_{p} \times \mathbf{r}_{p i}$.

\section{Comparison with another method}

The rotation averaging method presented in Guo and Zhao [15] and Zhao and Guo [41], which is similar to the methods used in many other literatures [13,20,21], is applied on the DEM models above for comparison. Their method to obtain the accumulated average particle rotation $\theta$ for a RVE packing is defined as:

$\theta=\frac{1}{N_{p}} \sum_{N_{p}} \theta_{p}$ 


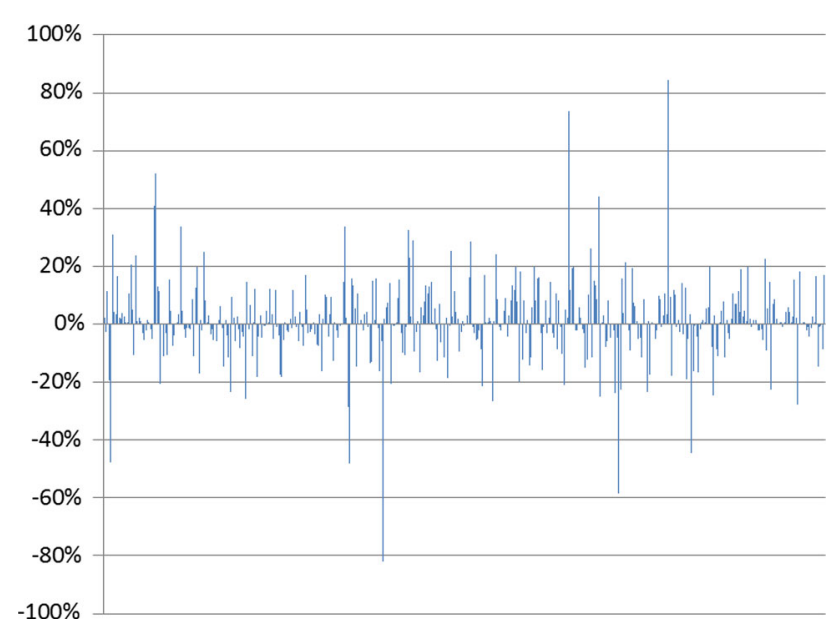

Fig. 14 The percentages of rotational velocities depending on $\omega_{p} \times \mathbf{r}_{p i}$ where $N_{p}$ is the number of particles, $\theta_{p}$ is the accumulated rotation of an individual particle.

The method is applied to the same locations in the DEM models as in the last section. Three different diameters for the averaging sphere is used: $4 d_{50}, 8 d_{50}$ and $12 d_{50}$, where $4 d_{50}$ is the mean grain diameter in the model. The results of rotations in y direction are shown in Figs. 15 and 16.

It can be seen that results are qualitatively similar to the results obtained by the general rotation averaging method presented in this paper, see Figs. 12 and 13. For both methods, the direction of averaging rotation agrees consistently with the inclination of the shear band, although the discrete particle rotation could be in different directions within shear bands. However, if we take a close look at the values of the averaged rotations, the method of Eq. (8) shows a scaledependency for both DEM models. The larger the averaging volume, the smaller averaged rotations. While the general

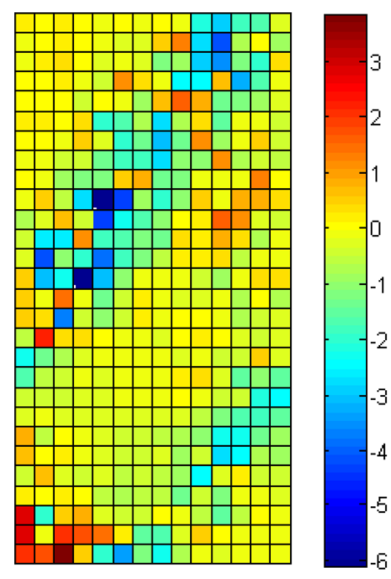

$[\mathrm{rad} / \mathrm{s}]$
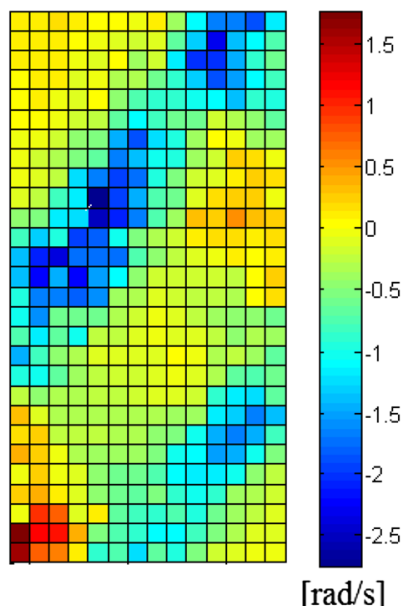

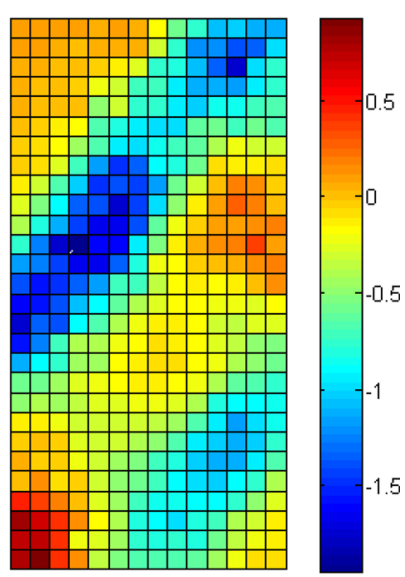

$[\mathrm{rad} / \mathrm{s}]$

Fig. 15 Averaging results with Eq. (8) for averaging diameters $4 d_{50}, 8 d_{50}$ and $12 d_{50}$, model with fixed boundary conditions
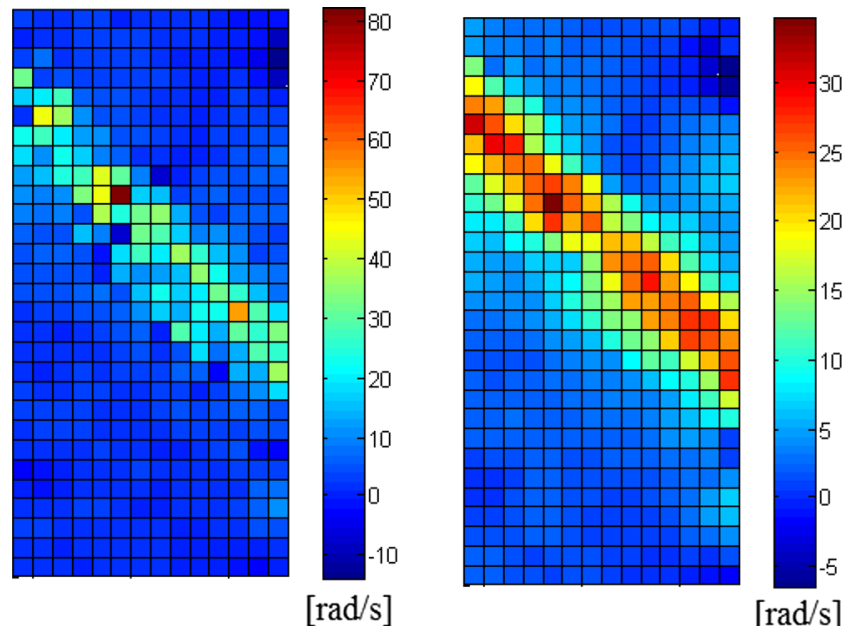

$[\mathrm{rad} / \mathrm{s}]$

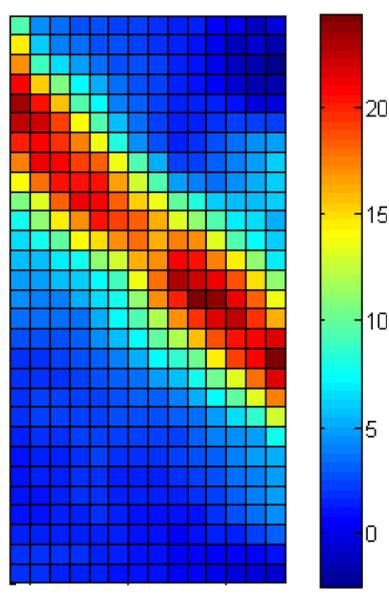

[rad/s]

Fig. 16 Averaging results with Eq. (8) for averaging diameters $4 d_{50}, 8 d_{50}$ and $12 d_{50}$, model with flexible boundary conditions 
rotation averaging method does not show scale-dependency for any of the DEM models. Also, the averaged rotations obtained by Eq. (8) are larger than those obtained by the general rotation averaging method.

There are several differences between these two methods. The method of Eq. (8) calculates the averaged rotations of all particles in the averaging volumes, but it does not take the particle's size, position or velocity into account. The general rotation averaging method only considers the particles in the boundary, but since all the particles have interactions in between, the behaviors of boundary particles depend very much on the particles inside the averaging volume. By taking the particle velocities into account, the vorticity of the particle velocities is considered in the general rotational averaging method. The method of Eq. (8) is performed on the RVE packing, therefore it does not need to define a rotational center. While the general rotation averaging method depends very much on the position of the rotational center.

\section{Size dependence of the method}

It is well known that the results of the averaging methods depend on the size of the averaging volume. To determine this relationship for the general rotation averaging method, we plot the averaged rotational velocity for three points in each DEM model with different averaging diameter. The ratio between the diameter of the averaging volume and the mean grain diameter $d_{a} / d_{50}$ range from 2 to 19 . The three points locate in the upper right corner $(4,33 \mathrm{~mm})$, the lower left corner $(18,5 \mathrm{~mm})$ and the middle $(10,20 \mathrm{~mm})$ of the center part of the model, see Fig. 10. For rigid boundary simulation, the results of averaged rotational velocities in $y$ direction are plotted in Fig. 17. For flexible boundary simulation, the results are plotted in Fig. 18.

It can be seen that the averaged rotational velocities vary a lot for $d_{a} / d_{50}<5$, while for $d_{a} / d_{50}>10$ the differences of averaged rotational velocities are not significant. For the points inside or close to a shear band (The first and second point in Fig. 17, the second and third point in Fig. 18), the averaged rotational velocity trends to converge to a nonzero value.

The aim of this paper is to present a unique averaging method which can be used for different scales. If microscale problems are considered $\left(d_{a}<10 d_{50}\right)$, the averaged rotation depends very much on the size of the averaging volume. For macroscale problems, the size of averaging volume $d_{a}=10 d_{50}$ would be a proper choice for calculation since the averaged results trend to reach a stable value for this averaging size. A circular shaped averaging volume might not always be the best choice, especially when the particles in
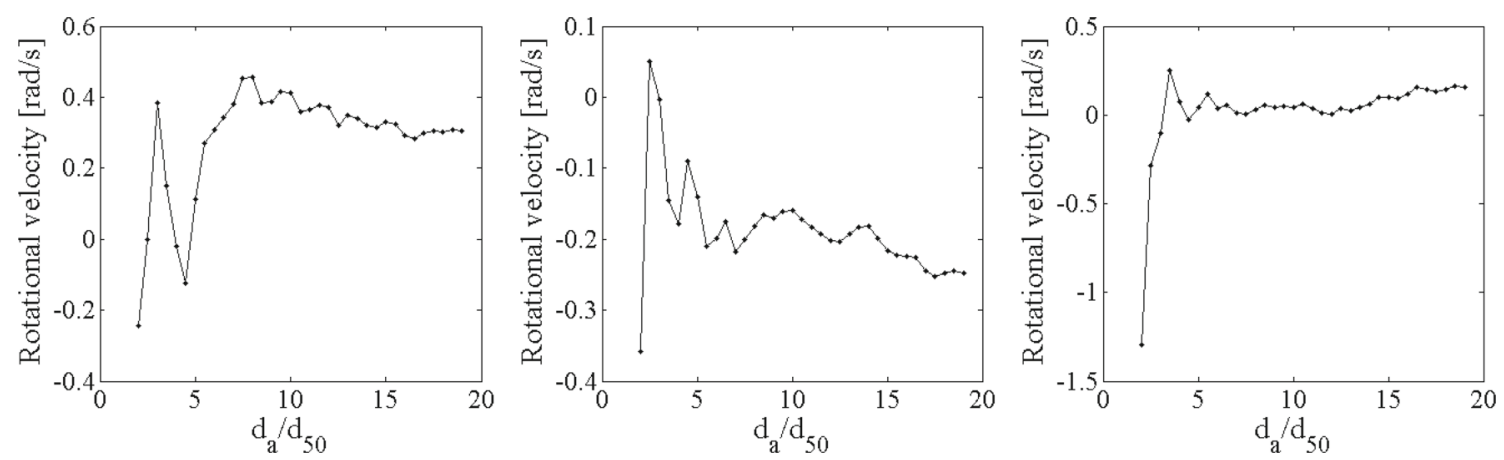

Fig. 17 Dependence of averaged rotational velocity in y direction on $d_{a} / d_{50}$ for three points in rigid boundary plane strain test
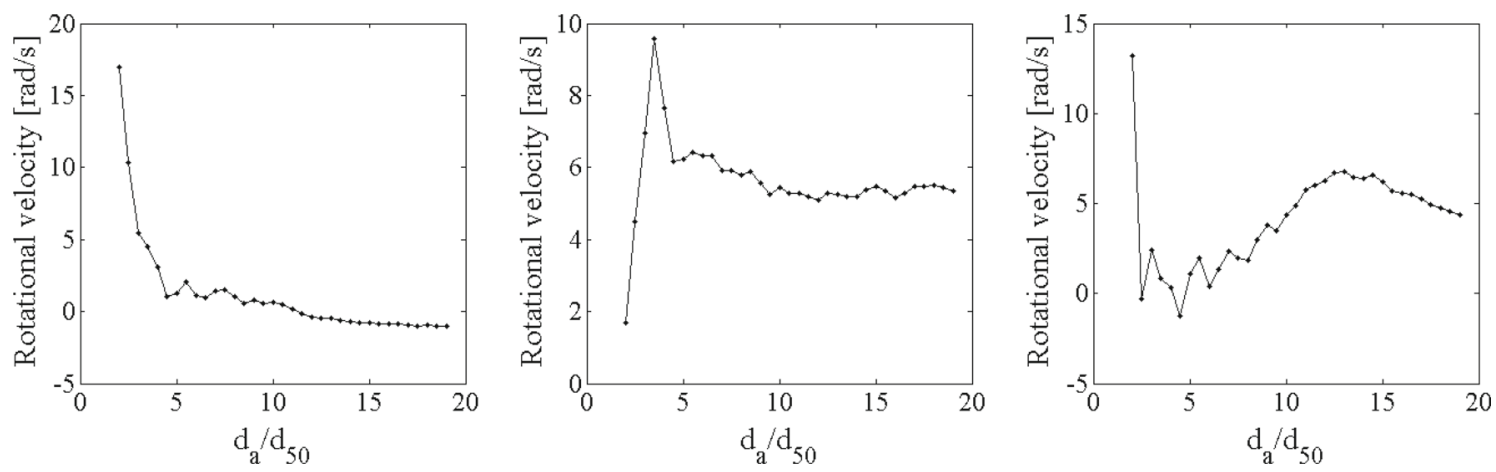

Fig. 18 Dependence of averaged rotational velocity in y direction on $d_{a} / d_{50}$ for three points in flexible boundary plane strain test 
the averaging circle have different behaviors. In these cases, the general rotation averaging methods need to be modified according to the shape of the averaging volume as mentioned in Sect. 2.

\section{Time averaging}

In order to see the change of averaged rotation over time, the averaged rotational speeds are measured with our averaging method in every 100 DEM steps. The measurement started from a time step in which the shear bands have been fully developed and the model undergoes a stable deformation. Therefore, the stability of the averaging method over time can be tested. Totally 11 measurements are taken. Two different diameters for the averaging volume are used $d_{a}=6 d_{50}$ and $d_{a}=10 d_{50}$. The averaging results for both DEM simulations inside and outside the shear band are shown in Figs. 19 and 20, in which the averaged results in all directions are given. For comparison, the same coordinate values are used for averaging sphere with $d_{a}=6 d_{50}$ and $d_{a}=10 d_{50}$ in the same position. However, the averaging results in different positions are shown with different coordinate values to make the plots easy to read.
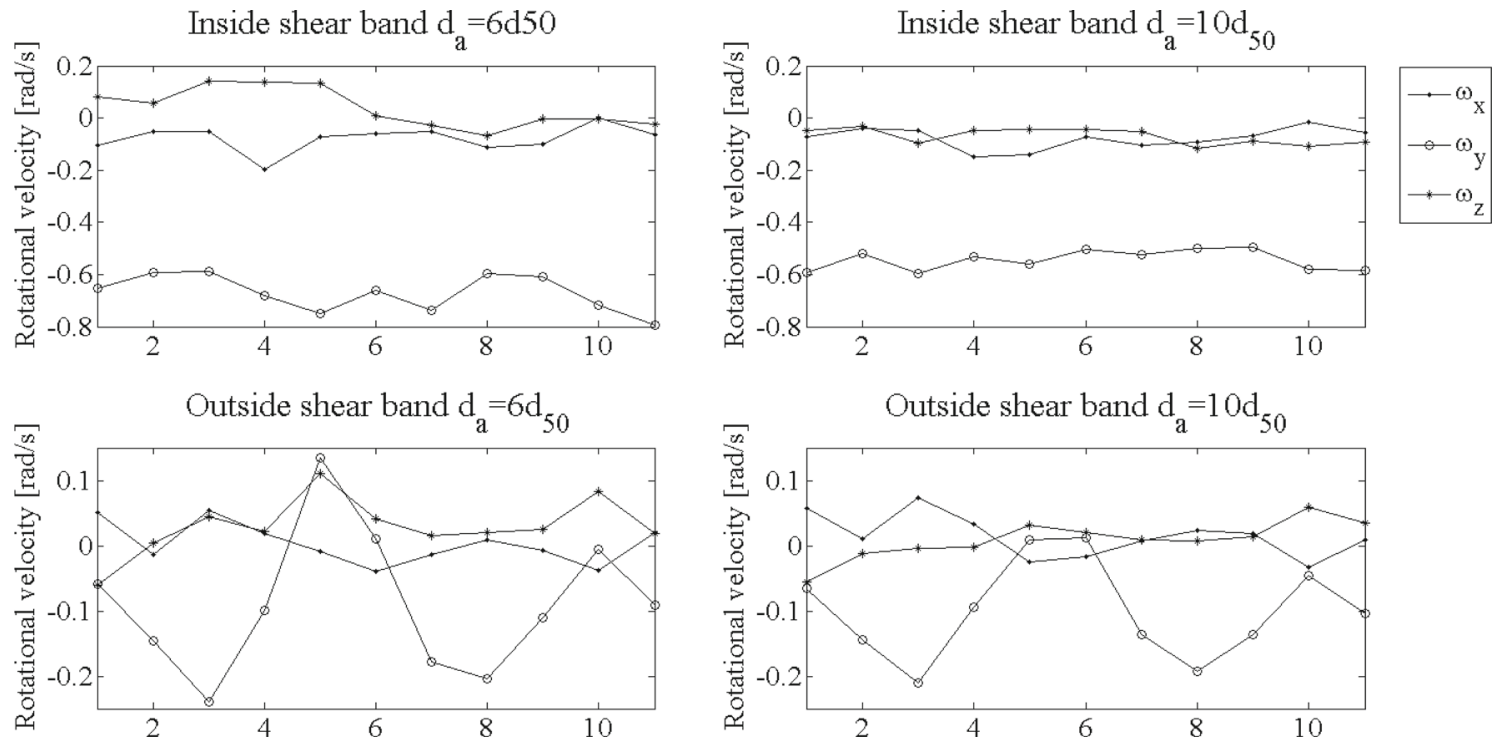

Fig. 19 Averaging results over time for DEM simulation with rigid stress boundary conditions
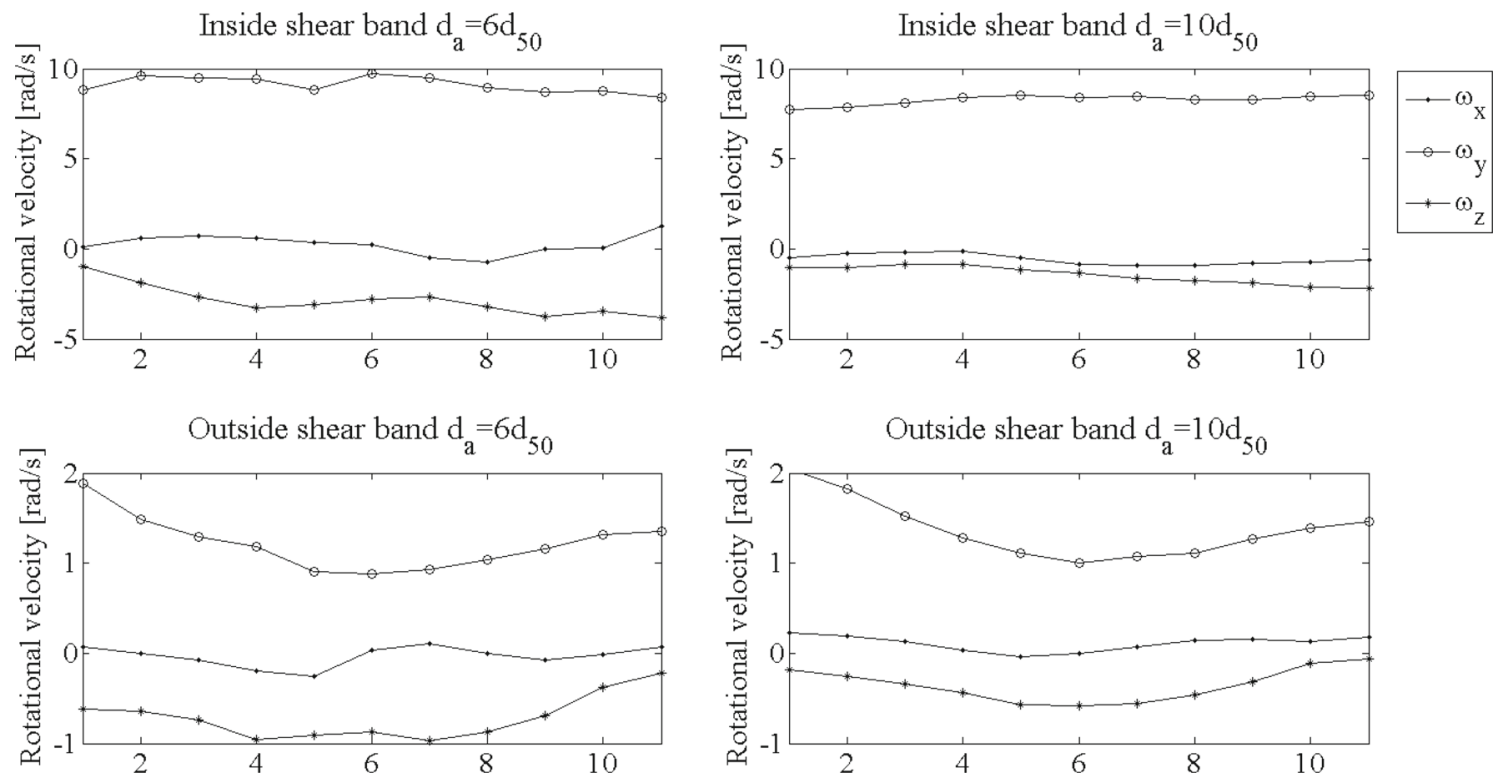

Fig. 20 Averaging results over time for DEM simulation with flexible stress boundary conditions 
It can be seen from the figures that the averaged rotations do not change very much over time, which means that this averaging method is relatively stable over time. Note that although the curves for rotations outside shear band in Fig. 19 show very large fluctuations, the change is actually not very large since the coordinate values on rotational velocity axis is very small in these two plots.

The time fluctuation for large averaging volume is less than for the small average volume. The reason can be that many rotations canceled out in large averaging volumes. Similar variations of the rotational speeds are given by averaging volumes of different sizes, which means that the method is stable by changes of averaging volume sizes. It can also be seen in the plots that the rotational speed in $\mathrm{x}$ and $\mathrm{z}$ direction $\left(\omega_{x}\right.$ and $\left.\omega_{z}\right)$ is almost zero, while $\omega_{y}$ can have large positive or negative values. This can be expected since plane strain conditions are considered here.

In the last section, it is already shown qualitatively that the rotational speed inside the shear band is larger than outside the shear band. From Figs. 19 and 20, we can calculate that the rotational speed inside the shear band is about 5 times larger than outside the shear band. The averaged rotations could be reduced by adding contact moments between balls.

\section{Conclusions}

A general rotation averaging method for granular materials in 2D and 3D cases are shown in this paper. Unlike conventional methods which require complicated averaging geometry and calculations, the new method is simple and has very low restrictions. In order to test the method, DEM simulations for plane strain tests with different boundary conditions are carried out. The rotation averaging method is applied to different parts of the model with different averaging volumes. The results show that the bulk rotational behavior of the DEM model can be well captured by the general rotation averaging method. The time averaging shows that the method is also very stable.

Acknowledgements Open access funding provided by University of Natural Resources and Life Sciences Vienna (BOKU). This work is supported by an EU funded Framework 7, Marie Curie Initial Training Network project PARDEM and university of natural resources and life sciences vienna. The authors would like to thank all the coworkers in PARDEM group. Our sincere thanks also goes to the software company Itasca, which offers us software support of PFC 3D.

\section{Compliance with ethical standards}

Conflict of interest All the authors acknowledge that this study contains original material, as a result of a purely academic study. Its publication has been approved by all coauthors and tacitly by the responsible authorities at the institutes where the work has been carried out.
Open Access This article is distributed under the terms of the Creative Commons Attribution 4.0 International License (http://creativecomm ons.org/licenses/by/4.0/), which permits unrestricted use, distribution, and reproduction in any medium, provided you give appropriate credit to the original author(s) and the source, provide a link to the Creative Commons license, and indicate if changes were made.

\section{References}

1. Altenbach, H., Eremeyev, V.A.: Strain rate tensors and constitutive equations of inelastic micropolar materials. Int. J. Plast 63, 3-17 (2014)

2. Bidier, S., Ehlers, W.: Particle-based modelling and computational homogenisation of granular media. In: 11th World Congress on Computational Mechanics, WCCM 2014, 5th European Conference on Computational Mechanics, ECCM 2014 and 6th European Conference on Computational Fluid Dynamics, ECFD 2014, pp. 3252-3263 (2014)

3. Chen, K.-C., Lan, J.-Y., Tai, Y.-C.: Description of local dilatancy and local rotation of granular assemblies by microstretch modeling. Int. J. Solids Struct. 46(21), 3882-3893 (2009)

4. Chung, Y.C., Ooi, J.Y.: Influence of discrete element model parameters on bulk behavior of a granular solid under confined compression. Part. Sci. Technol. 26(1), 83-96 (2008)

5. D’Addetta, G.A., Ramm, E., Diebels, S., Ehlers, W.: A particle center based homogenization strategy for granular assemblies. Eng. Comput. (Swansea, Wales) 21(2-4), 360-383 (2004)

6. Ebinger, T., Diebels, S., Steeb, H.: Numerical homogenization techniques applied to growth and remodelling phenomena. Comput. Mech. 39(6), 815-830 (2007)

7. Ehlers, W., Ramm, E., Diebels, S., D’ Addetta, G.A.: From particle ensembles to Cosserat continua: Homogenization of contact forces towards stresses and couple stresses. Int. J. Solids Struct. 40(24), 6681-6702 (2003)

8. Ehlers, W.: Recovering micropolar continua from particle mechanics by use of homogenisation strategies. Recent Developments and Innovative Applications, Computational Mechanics. Springer, Berlin (2011)

9. Froiio, F., Tomassetti, G., Vardoulakis, I.: Mechanics of granular materials: the discrete and the continuum descriptions juxtaposed. Int. J. Solids Struct. 43(25-26), 7684-7720 (2006)

10. Gitman, I.M., Askes, H., Sluys, L.J.: Representative volume: existence and size determination. Eng. Fract. Mech. 74(16), 2518-2534 (2007)

11. Goddard, J.D.: From granular matter to generalized continuum. Lect. Notes Math. 1937, 1-22 (2008)

12. Goldhirsch, I.: Stress, stress asymmetry and couple stress: from discrete particles to continuous fields. Granul. Matter 12(3), 239$252(2010)$

13. Göncü, F., Luding, S.: Effect of particle friction and polydispersity on the macroscopic stress-strain relations of granular materials. Acta Geotech. 8(6), 629-643 (2013)

14. Guo, N., Zhao, J.: A coupled FEM/DEM approach for hierarchical multiscale modelling of granular media. Int. J. Numer. Meth. Eng. 99(11), 789-818 (2014)

15. Guo, N., Zhao, J.: 3D multiscale modeling of strain localization in granular media. Comput. Geotechn. 80, 360-372 (2016)

16. Hirschberger, C.B., Sukumar, N., Steinmann, P.: Computational homogenization of material layers with micromorphic mesostructure. Philos. Mag. 88(30-32), 3603-3631 (2008)

17. Huang, W., Huang, L., Sheng, D., Sloan, S.W.: DEM modelling of shear localization in a plane Couette shear test of granular materials. Acta Geotech. 10(3), 389-397 (2015) 
18. Kozicki, J., Niedostatkiewicz, M., Tejchman, J., Muhlhaus, H.B.: Discrete modelling results of a direct shear test for granular materials versus FE results. Granul. Matter 15(5), 607-627 (2013)

19. Kruyt, N.P.: Statics and kinematics of discrete Cosserat-type granular materials. Int. J. Solids Struct. 40(3), 511-534 (2003)

20. Lätzel, M., Luding, S., Herrmann, H.J.: Macroscopic material properties from quasi-static, microscopic simulations of a twodimensional shear-cell. Granul. Matter 2(3), 123-135 (2000)

21. Lätzel, M., Luding, S., Herrmann, H.J.: From discontinuous models towards a continuum description. Continuous and Discontinuous Modelling of Cohesive-Frictional Materials. Springer, Berlin (2001)

22. Li, X., Yu, H.S., Li, X.S.: Macro-micro relations in granular mechanics. Int. J. Solids Struct. 46(25-26), 4331-4341 (2009)

23. Li, X., Li, X.S.: Micro-macro quantification of the internal structure of granular materials. J. Eng. Mech. 135(7), 641-656 (2009)

24. Li, X., Yu, H.S.: Numerical investigation of granular material behaviour under rotational shear. Geotechnique 60(5), 381-394 (2010)

25. Li, X., Liang, Y., Duan, Q., Schrefler, B.A., Du, Y.: A mixed finite element procedure of gradient Cosserat continuum for second-order computational homogenisation of granular materials. Comput. Mech. 54(5), 1331-1356 (2014)

26. Lin, J., Wu, W., Borja, R.I.: Micropolar hypoplasticity for persistent shear band in heterogeneous granular materials. Comput. Methods Appl. Mech. Eng. 289, 24-43 (2015)

27. Lin, J., Wu, W.: A comparative study between DEM and micropolar hypoplasticity. Powder Technol. 293, 121-129 (2016)

28. Lin, J., Wu, W.: Asymmetry of the stress tensor in granular materials. Powder Technol. 293, 113-120 (2016)

29. Meier, H.A., Steinmann, P., Kuhl, E.: On the multiscale computation of confined granular media. Comput. Methods Appl. Sci. 14, 121-133 (2009)

30. Miehe, C., Dettmar, J., Zäh, D.: Homogenization and two-scale simulations of granular materials for different microstructural constraints. Int. J. Numer. Meth. Eng. 83(8-9), 1206-1236 (2010)
31. Mühlhaus, H.-B., Vardoulakis, I.: Thickness of shear bands in granular materials. Géotechnique 37(3), 271-283 (1987)

32. Neff, P.: A finite-strain elastic-plastic Cosserat theory for polycrystals with grain rotations. Int. J. Eng. Sci. 44(8-9), 574-594 (2006)

33. Tejchman, J., Bauer, E.: Numerical simulation of shear band formation with a polar hypoplastic constitutive model. Comput. Geotech. 19(3), 221-244 (1996)

34. Tejchman, J., Bauer, E.: Modeling of a cyclic plane strain compression-extension test in granular bodies within a polar hypoplasticity. Granul. Matter 7(4), 227-242 (2005)

35. Tejchman, J., Górski, J.: Size effects in problems of footings on sand within micro-polar hypoplasticity. Arch. Hydroeng. Environ. Mech. 55(3-4), 95-124 (2008)

36. Tejchman, J., Wu, W.: FE-investigation of shear localization in granular bodies under high shear rate. Granul. Matter 11(2), 115128 (2009)

37. Weinhart, T., Thornton, A.R., Luding, S., Bokhove, O.: From discrete particles to continuum fields near a boundary. Granul. Matter 14(2), 289-294 (2012)

38. Weinhart, T., Labra, C., Luding, S., Ooi, J.Y.: Influence of coarsegraining parameters on the analysis of DEM simulations of silo flow. Powder Technol. 293, 138-148 (2016)

39. Wellmann, C., Lillie, C., Wriggers, P.: Homogenization of granular material modeled by a three-dimensional discrete element method. Comput. Geotech. 35(3), 394-405 (2008)

40. Wensrich, C.M.: Stress, stress-asymmetry and contact moments in granular matter. Granul. Matter 16(4), 597-608 (2014)

41. Zhao, J., Guo, N.: The interplay between anisotropy and strain localisation in granular soils: a multiscale insight. Géotechnique 65(8), 642-656 (2015)

42. Zhu, H.P., Hou, Q.F., Zhou, Z.Y., Yu, A.B.: Averaging method of particulate systems and its application to particle-fluid flow in a fluidized bed. Chin. Sci. Bull. 54(23), 4309-4317 (2009) 